01

\title{
Моделирование взаимодействия зонда магнитно-резонансного силового микроскопа с ферромагнитным образцом
}

\author{
(C) Р.В. Горев, Е.В. Скороходов, В.Л. Миронов
}

Институт фоизики микроструктур РАН, 603950 Нижний Новгород, Россия

e-mail: gorevrv@ipmras.ru

Поступило в Редакцию 28 марта 2019 г.

В окончательной редакции 28 марта 2019 г.

Принято к публикации 15 марта 2019 г.

Представлены алгоритм и результаты микромагнитного моделирования резонансного отклика зондового датчика (кантилевера) магнитно-резонансного силового микроскопа. Моделирование вынужденных колебаний намагниченности образца позволяет рассчитывать переменную составляющую силы, действующей на зонд со стороны образца, и строить спектры в виде зависимостей амплитуды колебаний кантилевера от напряженности внешнего магнитного поля. Моделирование временных зависимостей всех компонент поля намагниченности позволяет проводить анализ пространственных распределений спин-волновых резонансов образцов. Для тестовых объектов в виде прямоугольных пермаллоевых микрополосок получено хорошее согласие между модельными и экспериментальными спектрами.

Ключевые слова: магнитно-резонансная силовая микроскопия, ферромагнитный резонанс, спиновые волны, микромагнитное моделирование.

DOI: $10.21883 /$ JTF.2019.11.48322.126-19

\section{Введение}

Магнитно-резонансная силовая микроскопия (МРСМ) является высокочувствительным методом исследования локальных резонансных свойств магнитных систем с высоким пространственным разрешением [1-3]. В последнее время данный метод начал использоваться для исследований спин-волновых резонансов в ферромагнитных наноструктурах, применяемых в СВЧ микроэлектронике [4]. Однако одной из существенных проблем МРСМ является сопоставление наблюдаемых резонансов в регистрируемом МРСМ сигнале со спектром ферромагнитного резонанса (ФМР) в образцах с неоднородным распределением намагниченности. Это связано с тем, что взаимодействие зонда с образцом сильно зависит от взаимной ориентации магнитного момента зонда и внешнего магнитного поля, а также от взаимного расположения зонда и образца в пространстве. Кроме того, формирование МРСМ сигнала происходит в условиях влияния зонда на равновесное распределение намагниченности в ферромагнитном образце, что способствует возникновению целого ряда особенностей, которые сильно усложняют анализ МРСМ данных [5-10]. Все это делает необходимым использование численного моделирования для интерпретации результатов МРСМ измерений. Моделирование позволяет сопоставить наблюдаемые резонансные особенности в экспериментальном МРСМ спектре с соответствующими модами ФМР образца, а также учесть влияние магнитного поля зонда на пространственное распределение колебаний намагниченности и, следовательно, на спектр МРСМ сигнала $[8,9]$.
В настоящей работе на основе численного моделирования вынужденных колебаний намагниченности анализируется магнитостатическое взаимодействие в системе зонд-образец и рассчитывается отклик зондового датчика в модельном МРСМ эксперименте. Проводится сравнение экспериментального МРСМ спектра и результатов микромагнитного моделирования на примере тестового объекта в виде планарной ферромагнитной микрополоски прямоугольной формы.

\section{Взаимодействие зонда и образца}

В магнитно-резонансном силовом микроскопе для регистрации колебаний намагниченности образца используется датчик силы в виде упругой консоли с магнитной частицей (зондом) на конце (рис. 1). В общем случае магнитная сила, действующая на зонд со стороны образца, записывается следующим образом:

$$
\mathbf{F}=\nabla \int\left(\mathbf{M}_{p} \cdot \mathbf{H}\right) d V
$$

где $\mathbf{M}(\mathbf{r})$ - намагниченность зонда, $\mathbf{H}(\mathbf{r})$ - магнитное поле, создаваемое образцом в области зонда. Интегрирование проводится по объему зонда.

Для моделирования эффектов взаимодействия зонда с образцом широко используется простейшая модель зонда в виде однородно намагниченной сферы с магнитным моментом $\mu[11]$. Тогда $z$-компонента силы (1), которая 


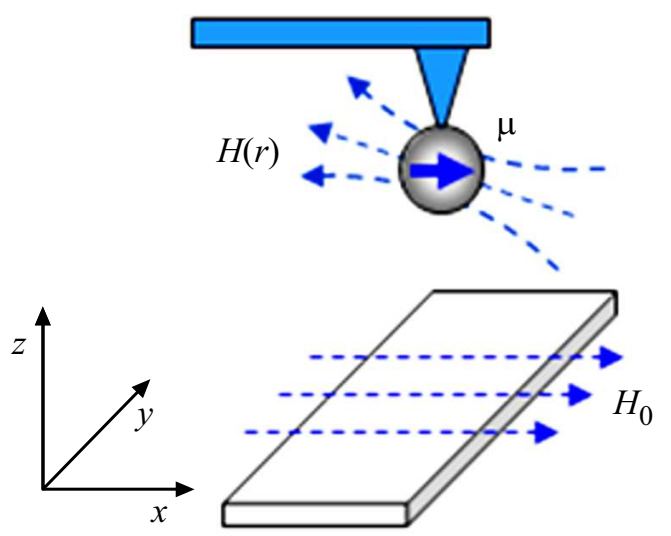

Рис. 1. Схематичное изображение системы зонд-образец.

обычно регистрируется в эксперименте, равна

$$
F_{z}=\mu_{x} \frac{\partial H_{x}}{\partial z}+\mu_{y} \frac{\partial H_{y}}{\partial z}+\mu_{z} \frac{\partial H_{z}}{\partial z},
$$

где градиент поля $\mathbf{H}(\mathbf{r})$ берется в центре сферы. Рассмотрим в качестве примера ситуацию, когда зонд имеет только $x$-компоненту магнитного момента (рис. 1). В этом случае выражение для силы упрощается

$$
F_{z}=\mu_{x} \frac{\partial H_{x}}{\partial z} .
$$

В МРСМ магнитный образец подвергается воздействию СВЧ излучения, амплитуда которого модулируется на частоте, равной резонансной частоте механических колебаний кантилевера. Под действием модулированной СВЧ накачки происходит возбуждение колебаний намагниченности образца. При этом на зонд действует периодическая сила, вызывающая колебания кантилевера, которые регистрируются детектором. Величина амплитуды колебаний кантилевера в МРСМ эксперименте $(\Delta)$ пропорциональна разности между силой, действующей на зонд при СВЧ накачке образца, и силой, действующей на зонд в отсутствие накачки:

$$
\Delta \propto \mu_{x}\left(\frac{\partial H_{1 x}}{\partial z}-\frac{\partial H_{2 x}}{\partial z}\right),
$$

где $\mathbf{H}_{1}$ - поле, создаваемое образцом на зонде в отсутствие накачки, $\mathbf{H}_{2}-$ поле от образца на зонде во время действия накачки.

\section{Тестовый эксперимент}

Нами были проведены тестовые измерения МРСМ спектра колебаний намагниченности одиночной микрополоски пермаллоя $\left(\mathrm{Ni}_{80} \mathrm{Fe}_{20}\right)$. Образец представлял собой массив пермаллоевых микрополосок размерами $3000 \times 500 \times 30 \mathrm{~nm}$, упорядоченных на прямоугольной решетке с периодом $10 \mu \mathrm{m}$ в направлении короткой оси микрополосок и $13 \mu \mathrm{m}$ в направлении длинной оси микрополосок. Измерения проводились с помощью МРСМ на базе зондового микроскопа „Solver HV“ $[12,13]$. В качестве зондового датчика использовался кантилевер NSG-1 (жесткость $0.01 \mathrm{~N} / \mathrm{m}$ и частота $10.5 \mathrm{kHz}$ ), на конец которого наклеивалась частица SmCo магнита диаметром $10 \mu \mathrm{m}$. Позиционирование зонда над образцом осуществлялось по АСМ изображению. Магнитный момент зонда был направлен вдоль поверхности образца, параллельно короткой оси микрополосок. Резонансная частота механических колебаний зондового датчика составляла $9.8 \mathrm{kHz}$, добротность 1000 . В эксперименте регистрировались зависимости амплитуды МРСМ отклика от величины намагничивающего поля (направленного также вдоль короткой оси микрополосок) при накачке образца микроволновым излучением с частотой $5.8 \mathrm{GHz}$ со $100 \%$ модуляцией по амплитуде на частоте $9.8 \mathrm{kHz}$. Поляризация магнитного СВЧ поля была линейной и направлена вдоль длинной стороны микрополосок. Экспериментальный МРСМ спектр, зарегистрированный при расположении зонда над центром одной из микрополосок, приведен на рис. 2. Расстояние от поверхности образца до центра зонда составляло $6 \mu \mathrm{m}$. В экспериментальном МРСМ спектре образца (рис. 2, кривая $A$ ) видны резонансы колебаний намагниченности микрополоски в виде провалов, отмеченных на рисунке цифрами $1-6$.

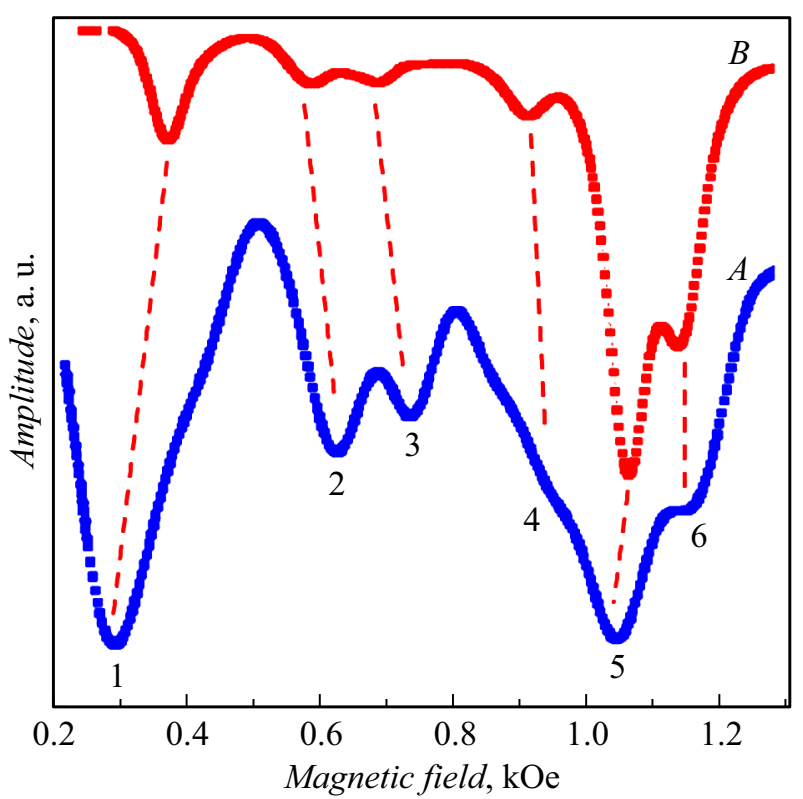

Рис. 2. МРСМ спектры пермаллоевой микрополоски. $A-$ экспериментальный МРСМ спектр, $B$ - модельный МРСМ спектр. Цифрами и пунктирными линиями отмечены резонансы колебаний намагниченности микрополоски. Экспериментальный и модельный МРСМ спектры отнормированы на максимум и сдвинуты друг относительно друга для удобства представления результатов. 


\section{Моделирование МРСМ отклика}

Микромагнитное моделирование МРСМ отклика проводилось с помощью программного пакета Object Oriented MicroMagnetic Framework (OOMMF) [14]. В качестве моделируемого образца была выбрана одиночная прямоугольная микрополоска из пермаллоя с размерами $3000 \times 500 \times 30 \mathrm{~nm}$ по аналогии с экспериментом. В расчетах использовались следующие материальные параметры пермаллоя: намагниченность насыщения $8 \cdot 10^{5} \mathrm{~A} / \mathrm{m}$, постоянная обменного взаимодействия $8.4 \cdot 10^{-12} \mathrm{~J} / \mathrm{m}$, безразмерный параметр диссипации 0.01. При моделировании полоска разбивалась на элементарные ячейки размером $10 \times 10 \times 30 \mathrm{~nm}$.

Алгоритм расчета МРСМ спектра включает следующие этапы:

1) к образцу прикладывается внешнее магнитное поле, после чего система релаксирует в состояние равновесия;

2) к системе прикладывается внешнее переменное поле с амплитудой $0.1 \mathrm{mT}$ на частоте $5.8 \mathrm{GHz}$ (соответствует экспериментальной ситуации);

3) далее следует процесс установления вынужденных колебаний намагниченности (установление колебаний в пермаллое заведомо наступает после 200 периодов);

4) пространственные распределения намагниченности для последних 5 периодов записываются в файл данных. Шаг по времени при оцифровке данных составляет 0.05 периода колебаний;

5) файлы данных из OOMMF экспортируются в пакет Mathematica, где распределения намагниченности усредняются по 5 периодам, т.е. рассчитывается пространственное распределение постоянной компоненты намагниченности в условиях СВЧ накачки;

6) далее в пакете Mathematica рассчитываются производные поля в центре зонда от равновесной намагниченности системы и от постоянной компоненты намагниченности системы при наличии внешнего переменного поля. Разность этих производных пропорциональна МРСМ сигналу (формула (4)).

Этапы 1-6 проводятся для различных значений внешнего магнитного поля с шагом $0.1 \mathrm{mT}$. МРСМ спектр представляет собой зависимость амплитуды силы, действующей на зонд, от внешнего магнитного поля.

В численном эксперименте моделировалась зависимость амплитуды силы, действующей на зонд, от величины внешнего подмагничивающего поля $\mathbf{H}_{0}$, направленного вдоль короткой оси микрополоски (ось $x$ ). Зонд имел магнитный момент $3.9 \cdot 10^{-11} \mathrm{~A} / \mathrm{m}^{2}$, направленный вдоль оси $x$. Расстояние от поверхности образца до центра зонда составляло $6 \mu \mathrm{m}$.

\section{Результаты и обсуждение}

Модельный МРСМ спектр микрополоски пермаллоя приведен на рис. 2 (кривая $B$ ). В данном спектре также наблюдаются 6 характерных провалов, соответствующих
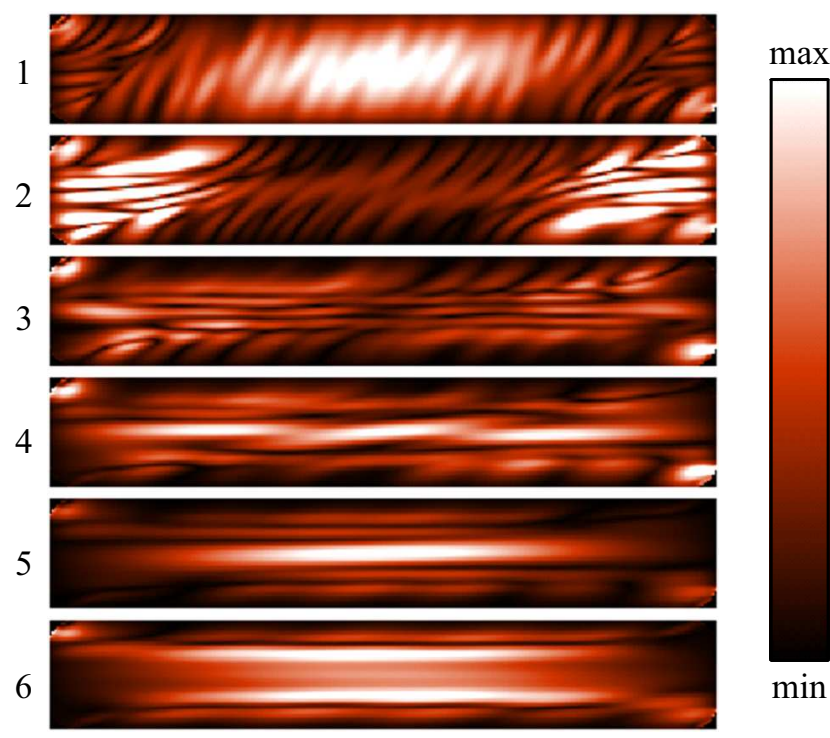

Рис. 3. Пространственные распределения амплитуд резонансных колебаний намагниченности пермаллоевой микрополоски, соответствующие резонансам 1-6 в МРСМ спектре.

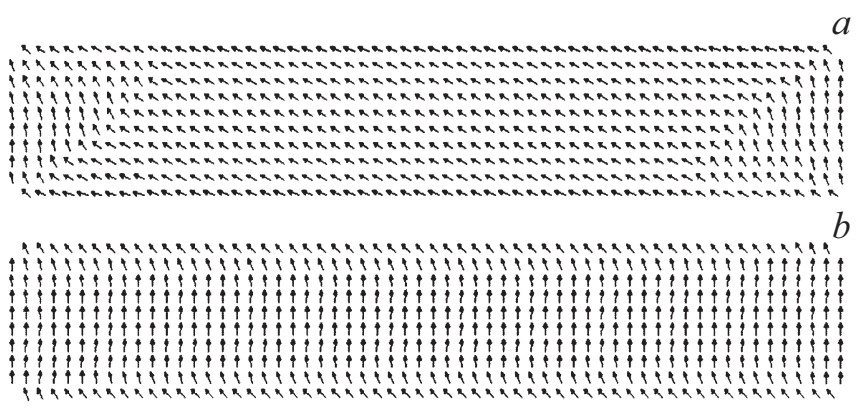

Рис. 4. Пространственные распределения намагниченности микрополоски в магнитном поле, направленном вдоль короткой оси; $a$ - поле соответствует резонансу $2 ; b-$ поле соответствует резонансу 6 .

резонансам колебаний намагниченности микрополоски. Суммарная сила, действующая на зонд, представляет собой сумму немагнитной и магнитной компонент [13]. Наличие провалов обусловлено тем, что магнитная компонента находится в противофазе с немагнитной компонентой. Как видно, наблюдается достаточно хорошая корреляция модельного и экспериментального МРСМ спектров. Различия между экспериментом и моделированием, по-видимому, обусловлены отклонением зонда от шарообразной формы и неоднородным распределением намагниченности в зонде.

Микромагнитное моделирование позволяет анализировать временные зависимости и пространственные распределения колебаний всех проекций $m_{x}, m_{y}, m_{z}$ переменной компоненты намагниченности. В частности, можно строить пространственные распределения амплитуды колебаний переменной составляющей намагничен- 
ности $[15]$

$$
m=\sqrt{m_{x}^{2}+m_{y}^{2}+m_{z}^{2}},
$$

которые позволяют визуализировать пространственную структуру резонансных мод. На рис. 3 приведены пространственные распределения амплитуды колебаний, соответствующие резонансам, отмеченным цифрами на рис. 2. Видно, что резонанс 1 представляет собой суперпозицию основной (квазиоднородной) моды стоячей магнитостатической волны и высокочастотной резонансной моды, обусловленной стоячей обменной волной [16]. Резонанс 2 соответствует неоднородным колебаниям намагниченности, локализованным в основном в углах микрополоски. Соответствующее распределение статической компоненты намагниченности микрополоски приведено на рис. 4, $a$. Резонансы 3-5 соответствуют суперпозиции различных мод стоячих спиновых волн, амплитуды которых меняются как вдоль длинной, так и вдоль короткой оси микрополоски. Резонанс 6 является локализованной краевой модой, связанной с неоднородностью размагничивающего поля образца [17-20]. В поле, соответствующем данному резонансу, микрополоска намагничена преимушественно вдоль короткой оси (рис. $4, b)$.

\section{Заключение}

Таким образом, в работе представлен алгоритм численного моделирования колебаний намагниченности и расчета МРСМ спектра ферромагнитного образца. Проведено сравнение модельного и экспериментального МРСМ спектров микрополоски из пермаллоя. Показано, что результаты расчетов МРСМ спектра хорошо коррелируют с результатами экспериментальных измерений. Это позволило провести анализ пространственной структуры резонансных мод микрополоски. Показано, что колебания намагниченности, соответствующие различным резонансам, имеют сложную структуру в виде суперпозиции стоячих магнитостатических и обменных волн, а также краевых мод, обусловленных неоднородным распределением намагниченности и внутреннего размагничивающего поля образца.

\section{Финансирование работы}

Работа выполнена в рамках государственного задания ИФМ РАН за 2019 г. (тема ГЗ 0030-2019-0021-С-01) и проекта РФФИ 18-02-00247.

\section{Конфликт интересов}

Авторы заявляют, что у них нет конфликта интересов.

\section{Список литературы}

[1] Sidles J.A. // Appl. Phys. Lett. 1991. V. 58, N. 24. P. 2854 2856. DOI: doi.org/10.1063/1.104757

[2] Rugar D., Budakian R., Mamin H.J., Chui B.W. // Nature. 2004. V. 430. P. 329-332. DOI: doi.org/10.1038/nature02658

[3] Degen C.L., Poggio M., Mamin H.J., Rettner C.T., Rugar D. // PNAS. 2009. V. 106, N. 5. P. 1313-1317. DOI: doi.org/10.1073/pnas.0812068106

[4] Nanomagnetism: Applications and Perspectives / Edited by Fermon C., Van de Voorde M. Wiley-VCH Verlag GmbH \& Co. Weinheim, Germany (2017).

[5] Mewes T., Kim J., Pelekhov D.V., Kakazei G.N., Wigen P.E., Batra S., Hammel P.C. // Phys. Rev. B. 2006. V. 74. N 14. P. 144424. DOI: doi.org/10.1103/PhysRevB.74.144424

[6] Chia H.-J., Guo F., Belova L.M., McMichael R.D. // Phys. Rev. B. 2012. Vol. 86. N 18. P. 184406.

DOI: doi.org/10.1103/PhysRevB.86.184406

[7] Hahn C., Naletov V.V., Loubens de G., Klein O., d'Allivy Kelly O., Anane A., Bernard R., Jacquet E., Bortolotti P., Cros V., Prieto J.L., Munoz M. // Appl. Phys. Lett. 2014. Vol. 104. N 15. P. 152410. DOI: doi.org/10.1063/1.4871516

[8] Volodin A., Van Haesendonck C., Skorokhodov E.V., Gorev R.V., Mironov V.L. // Appl. Phys. Lett. 2018. Vol. 113. N 12. P. 122407. DOI: doi.org/10.1063/1.5040072

[9] Горев Р.В., Миронов В.Л. // ФТТ. 2017. Т. 59. Вып. 11. C. 2154-2158. DOI: $10.21883 /$ FTT.2017.11.45053.10k

[10] Скороходов Е.В., Сапожников М.В., Горев Р.В., Володин А.П., Миронов В.Л. // ФТТ. 2018. Т. 60. Вып. 11. C. $2213-2218$. DOI: $10.21883 /$ FTT.2018.11.46665.25NN

[11] Джкексон Джс.. Классическая электродинамика / Пер. с англ. Г.В. Воскресенского и Л.С. Соловьева. Под ред. Э.Л. Бурштейна. М. Мир, 1965: 703 с.

[12] Скороходов Е.В., Сапожников М.В., Резник А.Н., Поляков В.В., Быков В.А., Володин А.П., Миронов В.Л. // ПТЭ. 2018. T. 5. C. $140-145$. DOI: $10.1134 / \mathrm{S} 0032816218040316$

[13] Скороходов Е.В., Сапожников М.В., Миронов В.Л. // Письма в ЖТФ. 2018. Т. 44. Вып. 5. С. 49-56. DOI: 10.21883/PJTF.2018.05.45707.17101

[14] Donahue M.J., Porter D.G. // Interagency Report NISTIR 6376. National Institute of Standards and Technology, Gaithersburg; http://math.nist.gov/oommf/

[15] Горев Р.В., Миронов В.Л., Скороходов Е.В. // Поверхность. Рентгеновские, синхротронные и нейтронные исследования. 2016. Т. 3. С. 37-40.

[16] Skorohodov E.V., Gorev R.V., Yakubov R.R., Demidov E.S., Khivintsev Yu.V., Filimonov Yu.A., Mironov V.L. // J. Magn. and Magn. Mater. 2017. Vol. 424. P. 118-121. DOI: doi.org/10.1016/j.jmmm.2016.10.024

[17] Bailleul M., Olligs D., Fermon C. // Phys. Rev. Lett. 2003. Vol. 91. P. 137204. DOI: doi.org/10.1103/PhysRevLett.91.137204

[18] McMichael R.D., Maranville B.B. // Phys. Rev. B. 2006. Vol. 74. P. 024424. DOI: doi.org/10.1103/PhysRevB.74.024424

[19] Wen-Bing C., Man-Gui H., Hao Z., Yu O., Long-Jiang D. // Chin. Phys. B. 2010. Vol. 19. N 8. P. 087502. DOI: doi.org/10.1088/1674-1056/19/8/087502

[20] Buess M., Haug T., Scheinfein M., Back C. // Phys. Rev. Lett. 2005. Vol. 94. P. 127205. DOI: 10.1103/PhysRevLett.94.127205 